\title{
Zinc Oxide Sub-Wavelength Structures as Antireflective Layer for Crystalline Silicon Solar Cells
}

\author{
Zhaoyuan Song ${ }^{1}$, Weijian Feng ${ }^{1}$, Yuee Chen $^{2}$, Guibin Yao ${ }^{1}$, Leilei Zhang ${ }^{1}$, \\ Lei Zhang ${ }^{1}$ and Wen Long ${ }^{1}$ \\ ${ }^{1}$ College of Science, Liaoning Shihua University, Liaoning Province, China \\ zysong815@163.com \\ ${ }^{2}$ College of Science, Yanshan University, Hebei Province, China
}

\begin{abstract}
The surface reflectance spectra of the zinc oxide $(\mathrm{ZnO})$ sub-wavelength structures (SWSs) are investigated by using the rigorous coupled wave theory. And the effective reflectance of $\mathrm{ZnO}$ SWS is calculated for the standard solar spectrum over the wavelength range from $400 \mathrm{~nm}$ to $1000 \mathrm{~nm}$. It is found that a lowest effective reflectance of $0.62 \%$ can be obtained for the optimized $\mathrm{ZnO}$ SWS, which is much less than that (5.94\%) of an optimized $\mathrm{ZnO}$ single layer antireflection coatings $(80 \mathrm{~nm})$. A 10.0- $\mu \mathrm{m}$-thick c-Si solar cell efficiency with the optimized ZnO SWS exhibits a 1.03\% improvement than that of $\mathrm{ZnO}$ single layer antireflection coatings. It is demonstrated that lower reflectance of the $\mathrm{ZnO}$ SWS across solar spectrum has potential application in solar cell industry.
\end{abstract}

Keywords: $\mathrm{ZnO}$, sub-wavelength structures, antireflection coatings, rigorous coupled wave theory, crystalline Si solar cells

\section{Introduction}

The Fresnel reflection of incident light is always existent on an interface between two different transparent media. A beam of light from air impinges on the surface of high index of refraction crystalline $\mathrm{Si}$, it will cause a great deal of photons to be reflected back from the interface. There is an effective method to reduce the number of lost photons by a substrate covered with single layer antireflection coating (SLARC) [1, 2]. However, it is not able to cover a wide range of the solar spectrum. In recent years, multilayer antireflection coatings have been studied which can fulfill wide wavelength range than SLARC [3-6]. Unfortunately, it is very difficult to associate with the different coating materials well and various physical-chemical properties will result in some problems, such as adhesion, thermal mismatch and the stability of the thin-film stack [7]. As is well known, the sub-wavelength structure (SWS) with feature size smaller than the incident wavelength is one of the best candidates to reduce the reflection of incident light in a broad range of the solar spectrum. Thus, some SWSs based on optical characteristic have been proposed for solar cells applications. For example, the effective reflectance of pyramid-shaped $\mathrm{Si}_{3} \mathrm{~N}_{4} \mathrm{SWS}$ has been analyzed by using a rigorous coupled-wave approach over the wavelength range from $400 \mathrm{~nm}$ to $1000 \mathrm{~nm}$ [8]. And then, the effective reflectance of the $\mathrm{Si}_{3} \mathrm{~N}_{4} \mathrm{SWS}$, which comprise a $\mathrm{Si}_{3} \mathrm{~N}_{4}$ layer superimposed an etched $\mathrm{Si}_{3} \mathrm{~N}_{4}$ grating with the cross-section of the strip in triangular shape, has also been studied using finite element analysis [9]. The results show that the lowest effective reflectance of the optimized $\mathrm{Si}_{3} \mathrm{~N}_{4}$ SWS is $1.98 \%$. In addition, a reflectivity below $1 \%$ is observed from $300 \mathrm{~nm}$ to $1000 \mathrm{~nm}$ for Si pyramid-shaped SWS grating which is approximated by a stack of 8 or 16 layers of Si slabs [10]. Here, a new kind of zinc oxide (ZnO) SWSs are proposed for crystalline $\mathrm{Si}$ solar cells applications, which mainly contain an etched $\mathrm{ZnO}$ 
layer on the bottom of an superimposed $\mathrm{ZnO}$ grating with the cross-section of the strip in multilevel surface profile.

In this study, we numerically examine the reflection characters of $\mathrm{ZnO}$ SWSs, which are optimized to get the lowest effective reflectance. And then, The ZnO SWS with the lowest effective reflectance are applied to a $10.0-\mu \mathrm{m}$-thick c-Si solar cell for estimating the electrical characteristics of a p-n junction solar cell. Finally, the solar cell efficiencies for ZnO SWS, bare sillion and ZnO SLARC are also compared and discussed.

\section{Structure and Simulation}

The proposed schematic diagram of the three-dimensional (3D) ZnO SWS is shown in Figure 1, which is composed of an etched $\mathrm{ZnO}$ and a $\mathrm{ZnO}$ grating with the cross-section of the strip in multilevel surface profile. The multilevel grating constituted by a stack of 9 layers of $\mathrm{ZnO}$ slabs with identical height is unlimited extension of the $x$-axis direction and reduplicate periodicity in the $y$-axis direction. The calculation parameters are defined as the period $A$, the height $h$, the top width $a$, the bottom width $b$ of the etched $\mathrm{ZnO}$ grating and the thickness of the $\mathrm{ZnO}$ layer to be $t$. In order to examine conveniently the effect of different width intermediate layers in the $\mathrm{ZnO}$ SWS, here we assume the width difference between neighbouring layers is $d$. So, the numerical relations between $a$ and $b$ can be expressed as $b=a+8 d$.

AM 1.5

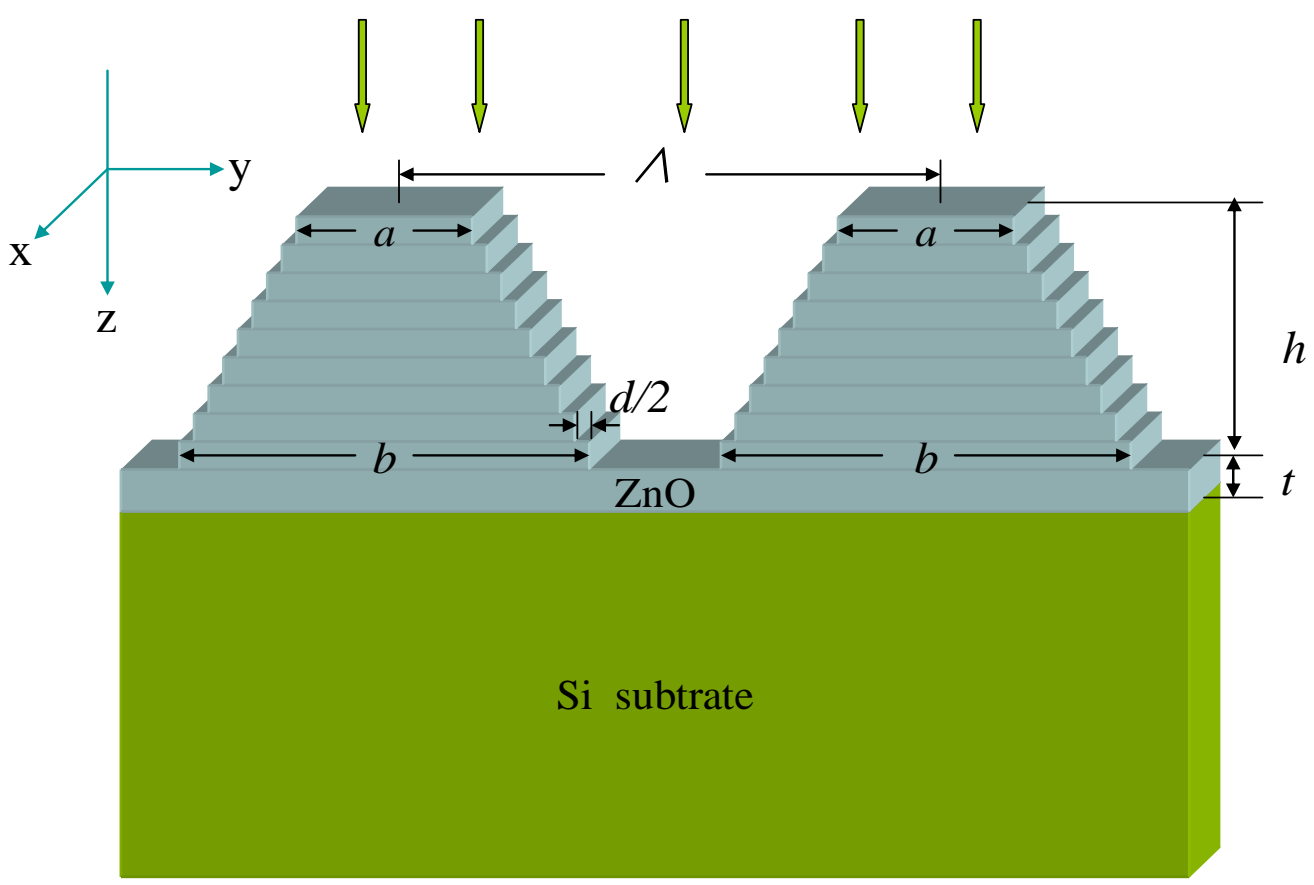

Figure 1.The Schematic Diagram of the Proposed ZnO SWS Structure, Where is the Period $\Lambda, H$ is the Height, $A$ is the Top Width, $B$ is the Bottom Width, $D$ is the Width Difference of Border Upon Layers of the Etched ZnO Grating and $S$ is the Thickness of the Zno Layer

The refractive index of $\mathrm{ZnO}$ in our model is the same with that used in [11]. In addition, an empirically fitted formula for the wavelength dependent of $n_{\mathrm{Si}}$ is employed in our simulation program [12]. 


$$
n_{\mathrm{Si}}=\sqrt{\sigma+\frac{A}{\lambda^{2}}+\frac{B \lambda_{1}^{2}}{\left(\lambda^{2}-\lambda_{1}^{2}\right)}}
$$

Where $\sigma=11.6858, A=9.39816 \times 10^{-12} \mathrm{~m}^{2}, B=8.10461 \times 10^{-3}$, and $\lambda=1.1071 \times 10^{-6} \mathrm{~m}$. The effective reflectance $\left(R_{\text {eff }}\right)$ for $\mathrm{ZnO}$ SWS is determined by the following equation [13].

$$
R_{\text {eff }}=\frac{\int_{\lambda_{1}}^{\lambda_{2}} R(\lambda) N_{\mathrm{ph}}(\lambda) \mathrm{d} \lambda}{\int_{\lambda_{1}}^{\lambda_{2}} N_{\mathrm{ph}}(\lambda) \mathrm{d} \lambda}
$$

Where, $\lambda$ is the light wavelength; $\lambda_{1}$ and $\lambda_{2}$ are the lower and upper wavelength of the spectrum range. Here, $\lambda_{1}=400 \mathrm{~nm}$ and $\lambda_{2}=1000 \mathrm{~nm}$ for the AM1.5 solar illumination; $R(\lambda)$ is the spectral reflectivity of the light; and $N_{\mathrm{ph}}(\lambda)$ is the photon number of the light with the wavelength $\lambda$ in the AM1.5 solar illumination [14].

When the sunlight, which can be approximated by a plane wave, is incident on the front surface of $\mathrm{ZnO}$ SWS, $R(\lambda)$ can be calculated by the rigorous coupled wave theory [15]. In addition, the incident light will propagate through the medium in a straight direction when the period of SWS is much less than the wavelength of the light [16]. Therefore, we merely study the reflectivity without considering the diffraction orders in this work. The initial parameter of $\mathrm{ZnO}$ SWS values in our simulation are summarized in Table 1.

Table 1. Some Initial Values for the Zno swS Parameters in the Simulation

\begin{tabular}{ll}
\hline Parameters & Value \\
\hline The grating period $\Lambda(\mathrm{nm})$ & 200 \\
The grating depth $h(\mathrm{~nm})$ & 150 \\
The thickness of the ZnO layer $t(\mathrm{~nm})$ & 70 \\
The bottom width of the grating $b(\mathrm{~nm})$ & 90 \\
The thickness of border upon layer $d(\mathrm{~nm})$ & 5 \\
\hline
\end{tabular}

\section{Result and Discussion}

\subsection{Surface Reflection}

Firstly, the surface reflectance of the ZnO SWS is calculated for several different heights $h$ as a function of incident wavelength over the wavelength range from $400 \mathrm{~nm}$ to $1000 \mathrm{~nm}$ in Figure 2. One can see that the reflectance is decreased with increasing the height of SWS in the whole visible region (e.g., a nearly flat curve at $h=135 \mathrm{~nm}$ ). On the other hand, the reflectance is undulate in creased with the increase of the wavelength when the height of $\mathrm{ZnO}$ SWS is over $135 \mathrm{~nm}$. And, the reflectance is increased with increasing the height for the wavelength ranging of $750 \mathrm{~nm}$ to $1000 \mathrm{~nm}$. Hence observations suggest that it is significant to discuss the proper height of $\mathrm{ZnO}$ SWS for the lowest reflectance. 


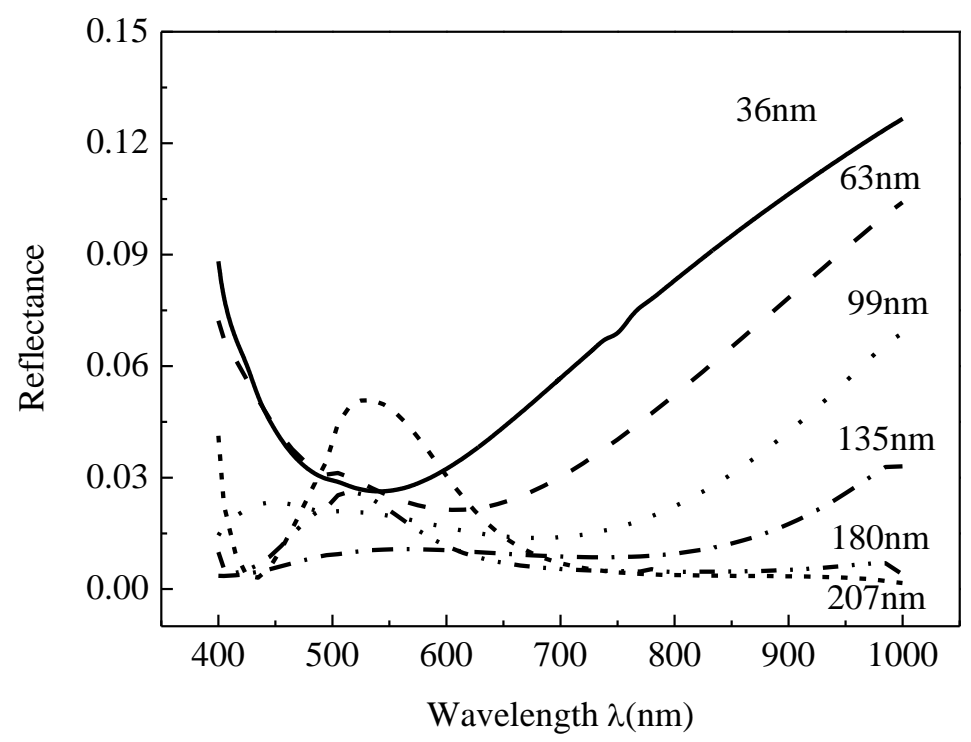

Figure 2. Calculated Reflectance of ZnO SWS for the Several Heights as a Function of Incident Wavelength

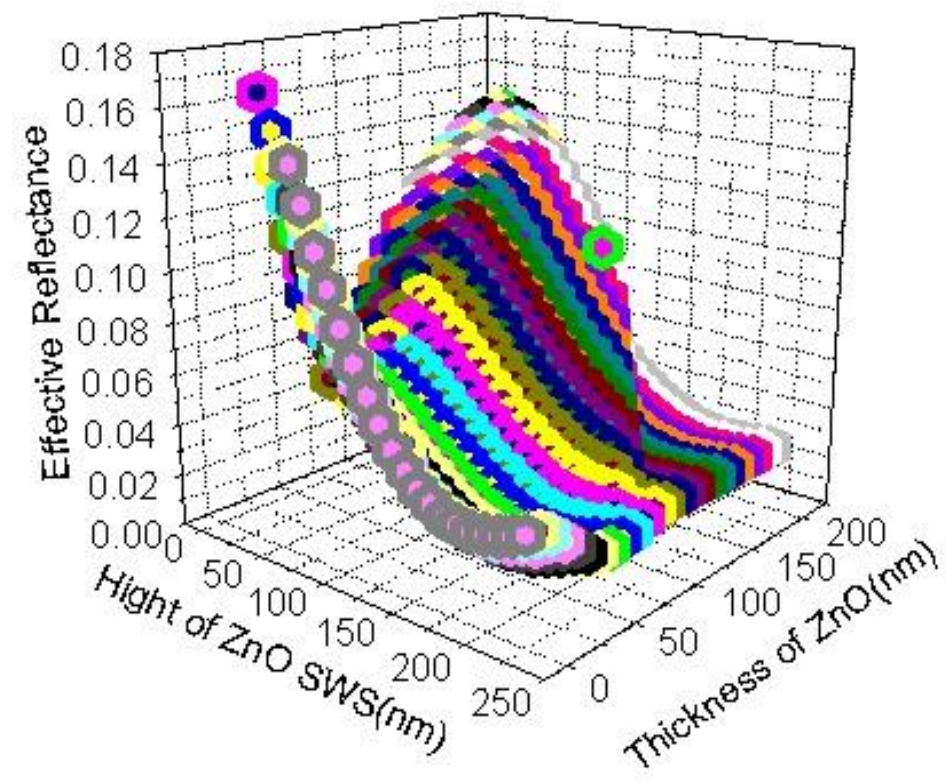
Figure 3. The Effective Reflectance Versus the Thickness of $\mathrm{ZnO}(S)$ and the
Height of ZnO SWS $(H)$

Thus, considering preferential the parameters for ZnO SWS in the $y$-axis direction, the effective reflectance of $\mathrm{ZnO}$ SWS is calculated for variety of $h$ and $s$, where other parameters from Table 1. The results is shown in Figure 3; from which one can see that the lowest of $R_{\text {eff }}=0.75 \%$ occurs at $h=171 \mathrm{~nm}$ and $t=65 \mathrm{~nm}$.

Figure 4 generalizes the lowest effective reflectance for the several $d$ as a function of $a$, where $h=171 \mathrm{~nm}, t=65 \mathrm{~nm}$ over the same wavelength range. It is shown that the effective reflectance firstly decreases and then increases as the increasing $a$, whatever the value of $d$. On the other hand, the larger $d$ (from $3 \mathrm{~nm}$ to $9 \mathrm{~nm}$ ), the smaller $a$ for the lowest effective reflectance. There is a minimum $R_{\text {eff }}(0.625 \%)$ for $a=55 \mathrm{~nm}$ and $d=5 \mathrm{~nm}$. And, it is obvious that $b=95 \mathrm{~nm}$ from the equation for previous description in this study. 


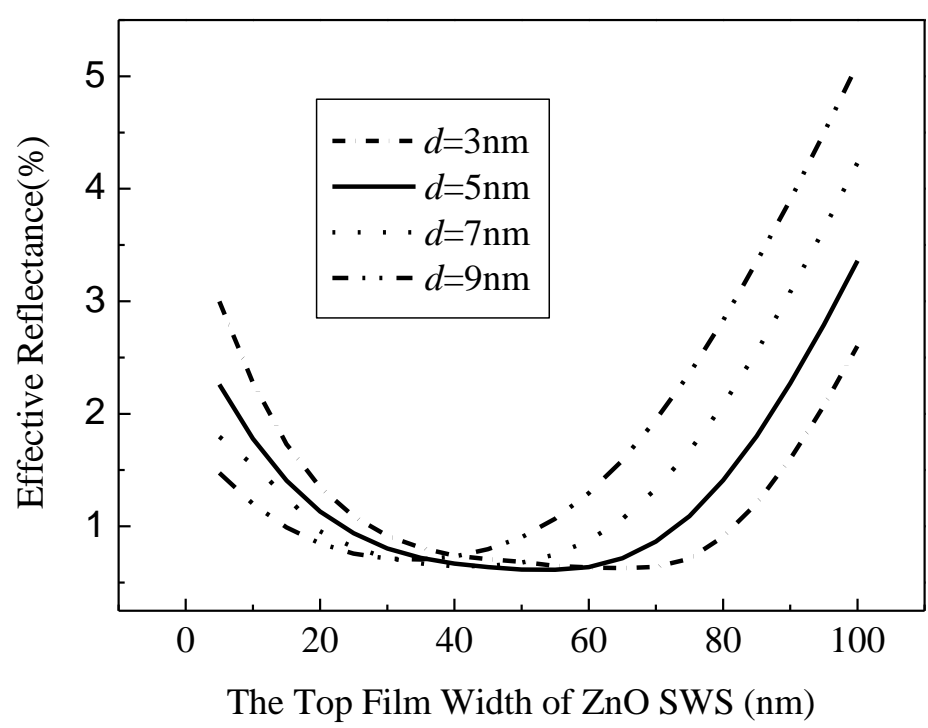

Figure 4. The Effective Reflectance for the Wavelength for the Varying to $1000 \mathrm{Nm}$ : Plot is As $A$ Function of $A$ and $D$ For ZnO SWS

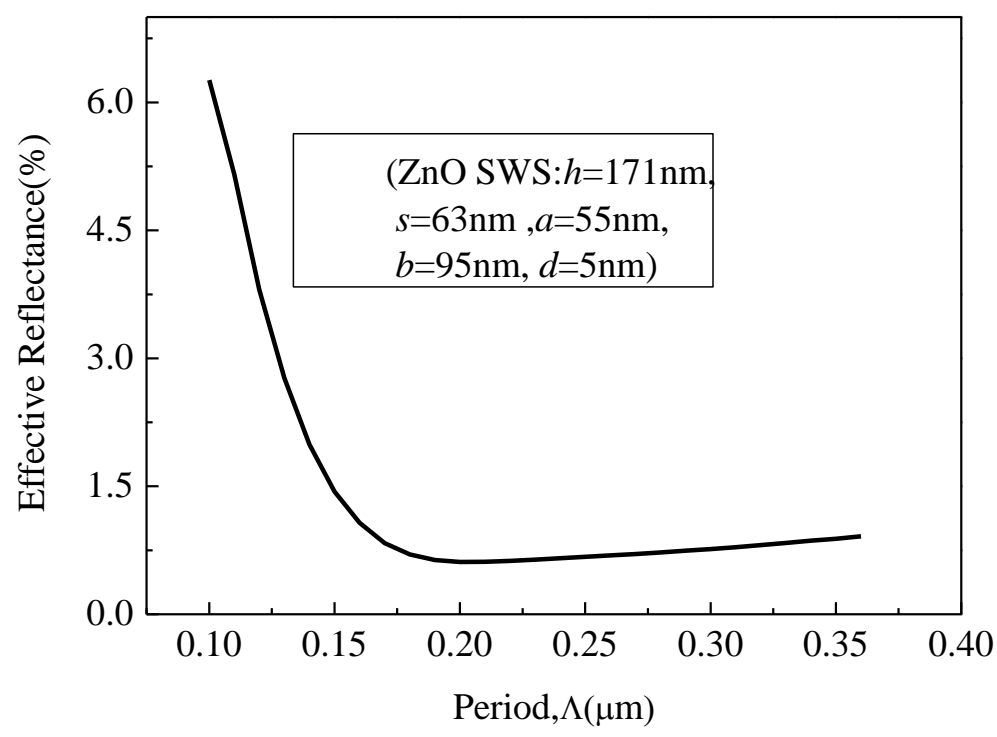

Figure 5. The Relation between the Effective Reflective $\boldsymbol{R}_{\mathrm{eff}}$ and $\Lambda$

We examine the effective reflectance of the $\mathrm{ZnO}$ SWS by varying $\Lambda$ which is equal to a unit cell width. In Figure 1 , the minimum value of $\Lambda$ is equal to the bottom width $b(95 \mathrm{~nm})$, so we change the unit cell width $\Lambda$ from $100 \mathrm{~nm}$ to 300 $\mathrm{nm}$ and fix the $h, t$ and $d$ at $171 \mathrm{~nm}, 65 \mathrm{~nm}$ and $5 \mathrm{~nm}$, respectively. The relation between the effective reflective and $\Lambda$ is shown in Figure 5 . It can be seen that the effective reflective drops intensely as $A$ from $100 \mathrm{~nm}$ to $150 \mathrm{~nm}$ and then varies a little increscence. The minimum of $R_{\text {eff }}$ is attained for $\Lambda=200 \mathrm{~nm}$.

In the end, the lowest effective reflectance of $R_{\text {eff }}=0.613 \%$ can be obtained with the optimized $\mathrm{ZnO}$ SWS, where $h=171 \mathrm{~nm}, t=65 \mathrm{~nm}, a=55 \mathrm{~nm}, d=5 \mathrm{~nm}$, and $\Lambda=200 \mathrm{~nm}$. The effective reflectance of the optimized ZnO SWS, bare sillion and 80 $\mathrm{nm} \mathrm{ZnO}$ SLARC in the wavelength ranging of $400 \mathrm{~nm}$ to $1000 \mathrm{~nm}$ are tabulated in Table 2. From Table 2, it is clear that the $\mathrm{ZnO}$ SWS has the lowest effective 
reflectance of $0.613 \%$ as compared to bare sillion, $80 \mathrm{~nm} \mathrm{ZnO} \mathrm{SLAR} \mathrm{coating.} \mathrm{The}$ spectral reflectivity of the optimized ZnO SWS, bare sillion and $80 \mathrm{~nm} \mathrm{ZnO} \mathrm{SLARC}$ over the wavelength ranging of $400 \mathrm{~nm}$ to $1000 \mathrm{~nm}$ are shown in Figure 6. It is found that the spectral reflectivity of $\mathrm{ZnO}$ SWS is lower than that of ZnO SLARC except a pimping section for the wavelength range of $600 \mathrm{~nm}$ to $700 \mathrm{~nm}$.

Table 2. Effective Reflectance for the Optimized Structures of ZnO SWS, Bare Sillion and $80 \mathrm{~nm}$ ZnO SLARC for the Wavelength Range from $400 \mathrm{~nm}$ to $1000 \mathrm{~nm}$

\begin{tabular}{cc}
\hline Structure & Effective reflectance $(\%)$ \\
\hline Bare Sillion & 21.21 \\
ZnO SLARC & 5.94 \\
ZnO SWS & 0.613 \\
\hline
\end{tabular}

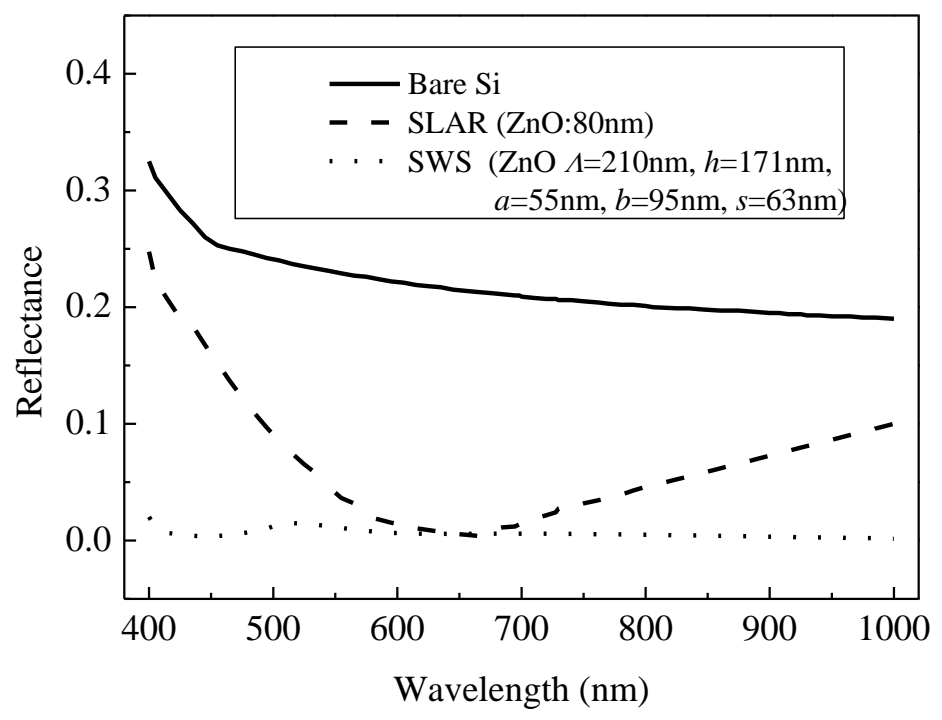

Figure 6. The Spectral Reflectivity of the Optimized ZnO SWS, ZnO SLARC and Bare Si

\subsection{The SWS for Solar Cell Applications}

As a example, a 10.0- $\mu$ m-thick c-Si solar cell model is applied to examine antireflective properties of zinc oxide sub-wavelength structure. The total efficiency of the solar cell can be calculated by:

$$
\eta=J_{\mathrm{sc}} V_{\mathrm{oc}} F F / P_{\text {in }}
$$

where $J_{\mathrm{SC}}\left(\mathrm{A} / \mathrm{cm}^{2}\right)$ is the short-circuit current density, $V_{\mathrm{OC}}(\mathrm{V})$ is the open-circuit voltage, $F F$ is the fill factor, and $P_{\text {in }}$ is defined as the incident power $\left(P_{\text {in }}=0.1 \mathrm{w} / \mathrm{cm}^{2}\right.$ under illumination AM 1.5).

$$
J_{\mathrm{SC}}=\int_{\lambda_{1}}^{\lambda_{2}} e \eta_{\mathrm{c}} A(\lambda) N_{\mathrm{ph}}(\lambda) \mathrm{d} \lambda
$$

where $e$ is the election charge, $A(\lambda)$ is the absorptance for each wavelength which can be calculated by RCWA [15]. The collection efficiency $\left(\eta_{\mathrm{c}}\right)$ of Si material is assumed to be a constant $85 \%$, which means that $85 \%$ of the incident light would be absorbed to generate electron-hole pairs [17].

$$
V_{\mathrm{oc}}=(k T / e) \ln \left(J_{\mathrm{sc}} / J_{\mathrm{so}}+1\right)
$$


Where $k$ is the Boltzmann's constant, $T=300 \mathrm{k}$ and $J_{\text {so }}$ is reserse bias saturation current $\left(1.5 \times 10^{-11} \mathrm{~A} / \mathrm{cm}^{2}\right)$. As for the fill factor, $F F=80 \%$ is that can be achieved with adequate choice of a load. In addition, the external quantum efficiency ( $E Q E)$ is calculated by [18]

$$
\operatorname{EQE}(\lambda)=A(\lambda) \eta_{\mathrm{c}}
$$

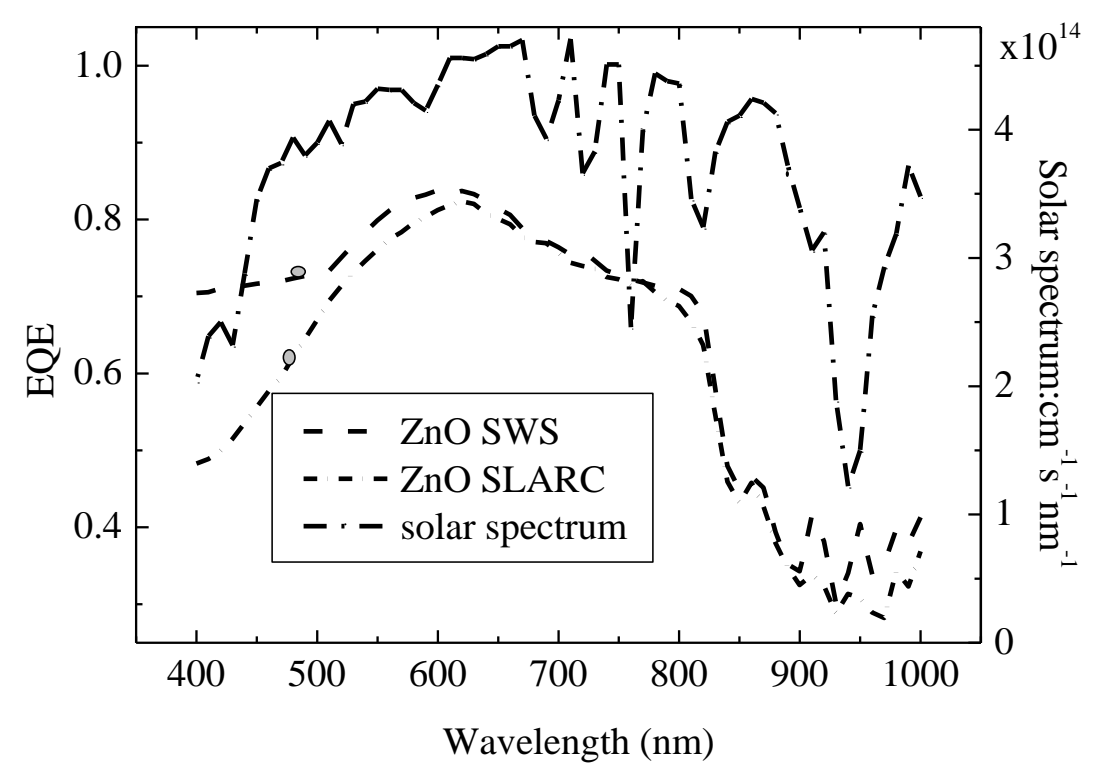

Figure 7. External Quantum Efficiency as a Function of Wavelength for 10$\mu \mathrm{m}$-Thick Si Solar Cell

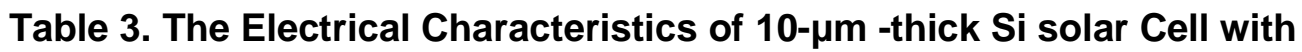
Different Surface Structures

\begin{tabular}{llll}
\hline Structure & $J_{\mathrm{sc}}\left(\mathrm{mA} / \mathrm{cm}^{-2}\right)$ & $V_{\mathrm{oc}}(\mathrm{mV})$ & Efficiency $(\%)$ \\
\hline Bare Sillion & 22.34 & 0.5471 & 9.77 \\
ZnO SLARC & 33.12 & 0.5568 & 14.75 \\
ZnO SWS & 35.38 & 0.5583 & 15.78 \\
\hline
\end{tabular}

The electrical characteristics of $10-\mu \mathrm{m}$-thick Si solar cell with different surface structures are shown in table 3. The solar cell efficiency with the optimized $\mathrm{ZnO}$ SWS exhibits a $1.03 \%$ improvement than that of $\mathrm{ZnO}$ single layer antireflection coatings. External quantum efficiency as a function of wavelength for $10-\mu \mathrm{m}$-thick $\mathrm{Si}$ solar cell is shown in Figure 7. The thick cell $(t=10 \mu \mathrm{m})$ of the $\mathrm{ZnO}$ SWS structure brings considerable absorption enhancement almost in the wide wavelength range of $400 \mathrm{~nm}$ to $1000 \mathrm{~nm}$.

\section{Conclusion}

In this paper, we have calculated the surface reflectance spectra of the indium tin oxide ( $\mathrm{ZnO})$ sub-wavelength structure (SWS). The effective reflectivity of $\mathrm{ZnO}$ SWS over the wavelength range from $400 \mathrm{~nm}$ to $1000 \mathrm{~nm}$ for standard solar spectrum is computed. Results of our study indicate that a lowest effective reflectivity of $0.613 \%$ can be obtained for the computed ZnO SWS with $h=171 \mathrm{~nm}, t=65 \mathrm{~nm}, a=55 \mathrm{~nm}, d=5 \mathrm{~nm}$ and $\Lambda=200 \mathrm{~nm}$, which is much less than those obtained an optimized $80 \mathrm{~nm} \mathrm{ZnO} \mathrm{SLARC} \mathrm{(5.94 \% ).} \mathrm{In}$ 
order to estimate the reflective effect of optimized ZnO SWS, a 10.0- $\mu \mathrm{m}$-thick c-Si solar cell efficiency based on the optimized $\mathrm{ZnO}$ SWS is also calculated, resulting an improvement of $1.03 \%$ efficiency than those of single layer antireflection coatings.

\section{Acknowledgment}

This work was supported by the Natural Science Foundation of China (No. 21403101), by the Foundation of Education Department of Liaoning Province (No. L2012135), by the Foundation of the Science and Technology Department of Liaoning Province (Nos. 2013020151 and 201602475) and by Program for the development of Science and Technology of Fushun city (Nos. 20153310 and 20141117). The authors also gratefully acknowledge the helpful comments and suggestions of the reviewers, which have improved the presentation.

\section{References}

[1] U. Schulz, "Review of modern techniques to generate antireflective properties on thermoplastic polymers”, Appl. Opt., vol. 45, no. 7, (2006), pp. 1608-1618.

[2] Z. C. Shen, W. J. Kong and S. H. Wang, "Graded index broadband antireflection coating prepared by glancing angle deposition for a high-power laser system", Chin. Phys. B, vol. 19, no. 4, (2010), pp. 044210.

[3] B. S. Richards, S. F. Rowlands and C. B. Honsberg, " $\mathrm{TiO}_{2}$ DLAR coatings for planar silicon solar cells", Prog. Photovolt: Res. Appl., vol. 11, no. 1, (2003), pp. 27-32.

[4] R. Sahraei, A. Daneshfar and A. Goudarzi, "Optical properties of nanocrystalline ZnS: Mn thin films prepared by chemical bath deposition method", Journal of Materials Science: Materials in Electronics, vol. 24, no. 1 , (2013), pp. 260-266.

[5] K. H. Mehdi and E. Mady, "Antireflective coatings: conventional stacking layers and ultrathin plasmonic metasurfaces, a mini-review", Materials, vol. 9, no. 6, (2016), pp. 9060497.

[6] C.-H. Chang, M.-H. Hsu and P.-C. Tseng, "Enhanced angular characteristics of indium tin oxide nanowhisker-coated silicon solar cells", Optics Express, vol. 19, no.10, (2011), pp. A219-A224.

[7] I. Suemune, "Enhanced light absorption in thin-film solar cells with light propagation direction conversion", Optics Express, vol. 21, no. S3, (2013), pp. A539-A547.

[8] K. C. Sahoo, Y. Li and E. Y. Chang, "Numerical calculation of the reflectance of sub-wavelength structures on silicon nitride for solar cell application", computer phys. Commun., vol. 180, no. 10, (2009), pp. 1721-1729.

[9] D. Craciun, G. Socol and S. L. Caër, "Gamma irradiation effects on the properties of indium zinc oxide thin films", Thin Solid Films, vol. 614, no. 9, (2016), pp. 2-6.

[10] H. Sai, H. Fujii and K. Arafune, "Antireflective subwavelength structures on crystalline Si fabricated using directly formed anodic porous alumina masks", Applied Physics Letters, vol. 88, no. 20, (2006), pp. 201116.

[11] M. K. Jebreel and M. E. Kordesch, "Optical constants and band edge of amorphous zinc oxide thin films", Thin Solid Films, vol. 515, no. 18, (2007), pp. 7393-7399.

[12] S. Khedim, A. Chiali, B. Benyoucefl, "Calculation of refractive index of some materials", Revue des Energies Renouvelables ICRESD-07 Tlemcen, (2007), pp. 337-341.

[13] D. N. Wright, E. S. Marsten and A. Holt, "Double layer anti-reflective coatings for silicon solar cells", Proceedings of the 31st IEEE Photovoltaic Specialists Conference (USA: Orlando), (2005), pp. 12371240.

[14] M. R. Mitroi, L. Fara and M. L. Ciurea, "Numerical procedure for optimizing dye-sensitized solar cells", Journal of Nanomaterials, vol. 2014, no. 1, (2014), pp. 1-6.

[15] L. Zhao, Y. H. Zuo and C. L. Zhou, "A highly efficient light-trapping structure for thin-film silicon solar cells", Solar Energy, vol. 84, no. 1, (2009), pp. 110-115.

[16] H. Sai, Y. Kanamori and K. Arafune, "Light trapping effect of submicron surface textures incrystalline Si solar cells”, Prog. Photovolt. Res. Appl., vol. 15, no. 15, (2007), pp. 415-423.

[17] R. S. Crandall, R. Williams and B. E. Tompkins, "Collection efficiency measurements on a- Si: H solar cells", J. Appl. Phys., vol. 50, no. 8, (1979), pp. 5506-5509.

[18] W. L. Bai, Q. Q. Gan and F. Bartoli, "Design of plasmonic back structures for efficiency enhancement of thin-film amorphous Si solar cells", Optic Letters, vol. 34, no. 23, (2009), pp. 3725-3727. 


\section{Authors}
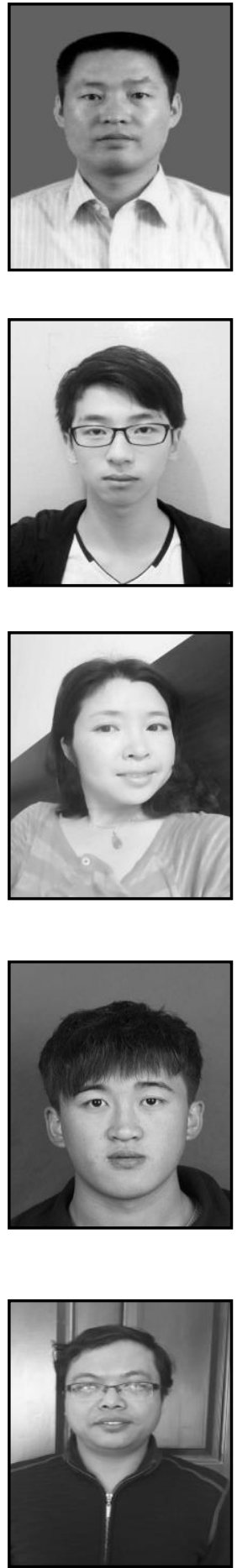

Zhaoyuan Song, he received the Ph.D. degree in optical engineering from Yanshan University, Qinhuangdao, China, in 2009. Since 2009, he works in College of Science, Liaoning Shihua University. His present research interests include solar cells and fuel cells. He is directing the Foundation of the Science and Technology Department of Liaoning Province on the development of new energy (No. 2013020151).

Weijian Feng, he is now studying for the B.S. degree in applied physics from Liaoning Shihua University, Fushun, China. He is now participating in the Foundation of the Science and Technology Department of Liaoning Province (No. 2013020151).

Yuee Chen, she received the Ph.D. degree in optical engineering from Yanshan University, Qinhuangdao, China, in 2009. Since 2009, she works in College of Science, Yanshan University. Her present research interests include solar cells and fuel cells. She is directing the Foundation of the Science and Technology Department of Heibei Province on the development of new energy (No. F2015203240) and the National Natural Science Foundation of China (No. 11504320).

Guibin Yao, his now studying for the B.S. degree in applied physics from Liaoning Shihua University, Fushun, China. He is now participating in the Foundation of the Science and Technology Department of Liaoning Province (No. 2013020151).

Leilei Zhang, he received the Ph.D. degree in condensed matter physics from Jilin University, China, in 2010. Since 2010, he works in College of Science, Liaoning Shihua University. His present research interests include solar cells and fuel cells. He is directing the Foundation of the Science and Technology Department of Liaoning Province on the development of new energy (No. 201602475), by Program for the development of Science and Technology of Fushun city (Nos.20153310 and 20141117) and by the National Natural Science Foundation of China (No.21403101). 

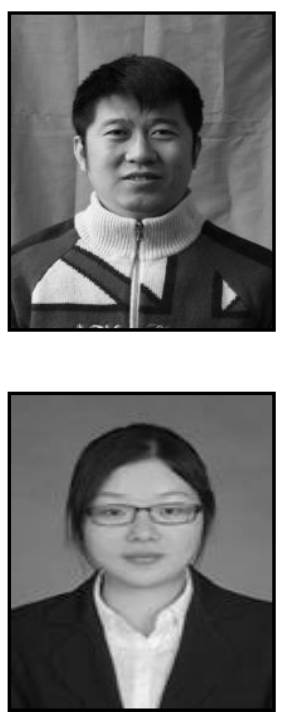

Lei Zhang, he is now studying for Material Physics and Chemistry Doctor Degree in Northeastern University and works in College of Science, Liaoning Shihua University. His present research fields are solid oxide fuel cells. He is now participating in the National Natural Science Foundation of China (No. 21403101) and the Foundation of the Science and Technology Department of Liaoning Province on the development of new energy (No. 2013020151).

Wen Long, she received the B.E. degree from Hebei Normal University of Science and Technology, Qinghuangdao, China, in 2009. She received the Ph.D. degree from Jilin University, Changchun, China, in 2014. She is currently working in Liaoning Shihua University. Her research interests is mainly focused on solid oxide fuel cell and new energy material and devices. 